\title{
Intensification of steam reforming of natural gas: Choosing combustible fuel and reforming catalyst
}

\author{
Georgios D. Stefanidis, Dionisios G. Vlachos* \\ Department of Chemical Engineering and Center for Catalytic Science and Technology (CCST), University of Delaware, 150 Academy Street, Newark, DE 19716, USA
}

\section{A R T I C L E I N F O}

\section{Article history:}

Received 8 July 2008

Received in revised form 27 January 2009

Accepted 2 June 2009

Available online 10 June 2009

\section{Keywords:}

Steam reforming

Catalytic combustion

Microreactors

Syngas

Hydrogen

Natural gas

Methane

Propane

Rhodium

Nickel

\begin{abstract}
A B S T R A C T
The steam reforming of methane in a parallel plate microreactor, consisting of alternating channels carrying out catalytic combustion and reforming on opposite sides of a wall, is modeled with fundamental kinetics and a pseudo-2D reactor model. It is shown that at high fuel conversions, the choice of hydrocarbon combustible fuel is immaterial when suitable compositions are used so that the energy input is kept the same. On the other hand, direct comparison of Rh and $\mathrm{Ni}$ indicates that the choice of reforming catalyst is critical. Speed up of heat transfer via miniaturization is insufficient for process intensification; catalyst-intensification is also needed to avoid hot spots and enable compact devices for portable and distributed power generation.
\end{abstract}

(c) 2009 Elsevier Ltd. All rights reserved.

\section{Introduction}

Intensifying fuel processors for syngas or hydrogen production is nowadays a rather pressing research topic due to the increasing demand for cleaner, safer, and more economical processes (Farrauto et al., 2003; Trimm and Onsan, 2001). Such processes may find several applications of every day life ranging from portable electronic devices, such as laptops and cell phones, to onboard power production in vehicles as well as distributed energy and gas-to-liquid (GTL) systems at local scale in urban or rural areas (e.g., house, building, or farm scale) and offshore. Contrary to the industrial scale, where process' economics dictate design, process intensification is challenging due to the need, among others, to match process conditions of a downscaled processor (e.g., different heat transfer/removal mechanisms, reduced residence times, etc.) (Norton et al., 2005; Vlachos, 2008).

Methane steam reforming (SR) is still the principal industrial method for natural syngas and hydrogen production (Farrauto et al., 2003). It has been studied in mesoscale reactors theoretically and experimentally (Mei et al., 2007; Robbins et al., 2003; Venkataraman et al., 2003; Yuan et al., 2007; Zanfir and Gavriilidis, 2003). In recent work (Stefanidis and Vlachos, 2008; Stefanidis et al., 2009), we

\footnotetext{
* Corresponding author. Tel.: +1 302831 2830; fax: +1 3028311048.

E-mail address: vlachos@udel.edu (D.G. Vlachos).
}

showed via extensive proof-of-concept simulations that SR on $\mathrm{Rh}$ at microscale (sub-millimeter characteristic length scale) and millisecond contact times is feasible and thus, syngas production can be intensified by hundred to thousand times. Our finding has independently been verified experimentally by Tonkovich et al. (2007). In Stefanidis and Vlachos (2008), Stefanidis et al. (2009), the simulations were presented for a catalytic plate reactor (CPR), where SR on $\mathrm{Rh}$ and propane combustion on Pt take place in alternate channels. The role of operating conditions, such as flow rates, inlet composition, and catalyst loading, and design parameters, such as the wall material and the channels size, were analyzed. Different operating lines were mapped out, and an operation strategy for variable power output was presented.

Herein we extend our work by exploring the role of the combustible fuel and the reforming catalyst. Regarding combustion, we compare methane and propane fuels. Methane is an obvious combustible fuel due to its availability in natural gas and the process simplification stemming from using a single fuel. On the other hand, propane exhibits enhanced stability (Norton and Vlachos, 2004), and natural gas is a mixture of small hydrocarbons. In integrated systems, one may envision burning leftover hydrogen from a fuel cell. Furthermore, reforming of ethanol coupled with combustion of ethanol may also become attractive in some locations/applications. Since the combustible fuel will most probably be a mixture of fuels, it is important to understand how the operating strategies may vary with the choice of combustible fuel. 


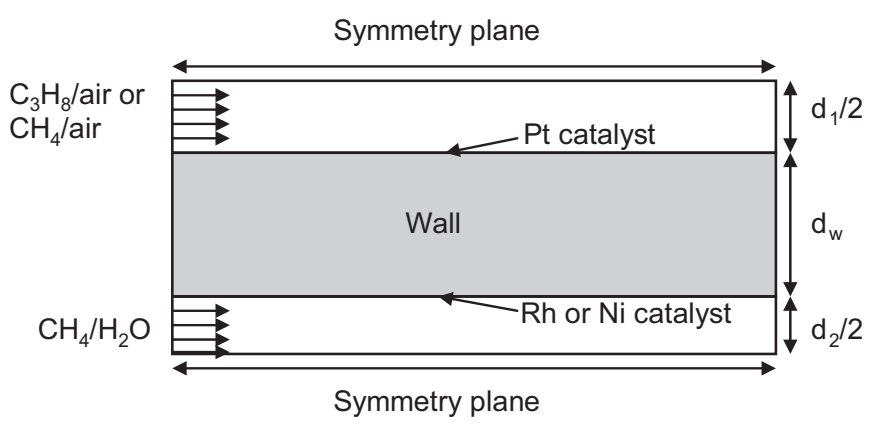

Fig. 1. Schematic of the simulated multifunctional microreactor.

Regarding SR, we compare Ni and $\mathrm{Rh}$. Ni is the industrial catalyst for hydrocarbon SR because is a robust, active catalyst and relatively tolerant to poisons, such as sulfur, chloride, and heavy metals. However, coking, although lower on Ni than on other transition metals of the fourth period, is a major issue industrially and requires high inlet steam-to-carbon ratios (S:C) $>3$ (Farrauto et al., 2003). Noble metals such $\mathrm{Ru}, \mathrm{Rh}$, and Pd are highly active and less prone to coking, allowing operation at lower $\mathrm{S}: \mathrm{C}$ ratios, which increase hydrogen yield. However, these are very expensive and sensitive to poisoning catalysts. Industrially, SR on Ni is heat transfer controlled. Herein, we explore whether $\mathrm{SR}$ on $\mathrm{Ni}$ is feasible by intensifying the process via miniaturizing.

\section{Model description}

\subsection{Reactor model}

A multifunctional, CPR microreactor combining a catalytic combustion channel and a SR channel, as shown in Fig. 1, is simulated. The two channels are separated by a solid wall, being catalytically coated on the two sides, which acts as a heat exchanger between the two processes. This is a relatively simple reactor concept, which offers the advantage of compactness, the possibility of scaling out the process by stacking up together many plates and the flexibility of using different configurations (co-current vs. counter-current flow) as well as different catalysts in the two channels. In this work, propane or methane combustion on Pt takes place on one side of the wall. Methane SR along with water-gas shift (WGS) on Rh or Ni take place on the other side of the wall. In the case of Ni catalyst, the reverse methanation (RM) reaction is also accounted for.

Only the co-current flow configuration is considered since it was found to provide better reaction zone overlap and hot-spot minimization (Deshmukh and Vlachos, 2005a, 2005b) in agreement with mesoscale systems (Kolios et al., 2005, 2007; Zanfir and Gavriilidis, 2003). The microreactor dimensions and the nominal operating conditions are quoted in Table 1 . The pseudo-2D reactor model presented in Kaisare et al. (2008), Kaisare and Vlachos (2007a, 2007b) is used. The model consists of the mass and the energy balances for the gas and the solid phase in the axial direction and transverse heat and mass transfer through lumped transport coefficients. The latter depend on the Nu and Sh numbers, which vary non-monotonically along the reactor length (Kaisare et al., 2008; Norton and Vlachos, 2004). Following previous work, we use average constant values of 4 and 3.8 for the Nu and Sh number, respectively, which ensure good quantitative agreement between the commercial CFD code (FLUENT, 2004) and our pseudo-2D code (Kaisare et al., 2008; Stefanidis et al., 2008). The reactor length is discretized with 400 equidistant nodes.
Table 1

Nominal values of simulation variables that are kept constant unless otherwise mentioned.

\begin{tabular}{lll}
\hline Reactor length & $l$ & $5 \mathrm{~cm}$ \\
Wall thickness & $d_{w}$ & $750 \mu \mathrm{m}$ \\
Wall conductivity & $k_{s}$ & $100 \mathrm{~W} / \mathrm{m} \mathrm{K}$ \\
& & \\
Combustion channel & $d_{1}$ & \\
$\quad$ Gap size & $T_{\text {in }}$ & $300 \mu \mathrm{m}$ \\
Inlet temperature & $P$ & $300 \mathrm{~K}$ \\
Pressure & $\mathrm{SAF}$ & $1 \mathrm{~atm}$ \\
Catalyst to geometric surface area & & 1.7 \\
& & \\
Steam reforming channel & $d_{2}$ & $200 \mu \mathrm{m}$ \\
Gap size & $\mathrm{S} / \mathrm{C}$ & 2 \\
Inlet composition & $T_{\text {in }}$ & $400 \mathrm{~K}$ \\
Inlet temperature & $P$ & $1 \mathrm{~atm}$ \\
Pressure & $\mathrm{SAF}$ & 1.0 \\
Catalyst to geometric surface area & & \\
\hline
\end{tabular}

\subsection{Kinetic models}

SR and WGS on Rh are modeled as in Stefanidis and Vlachos (2008), Stefanidis et al. (2009) with lumped, reversible rate expressions recently derived in Maestri et al. (2008). The lumped model is a product of a posteriori reduction of the detail microkinetic model of Maestri et al. (2009), which is an updated version of the earlier model of Mhadeshwar and Vlachos (2005) using a comprehensive experimental data set with over 300 experimental points and multiple processes, e.g., partial oxidation, steam and dry reforming, WGS, reverse WGS, and combustion (conditions: $T=300-850^{\circ} \mathrm{C}, \mathrm{CH}_{4}: \mathrm{H}_{2} \mathrm{O}=1.5-2.5$ in the case of SR) from Donazzi et al. (2008a, 2008b). Contrary to power-law fitted models or postulated chemical pathways and a rate-determining step (e.g. the Langmuir-Hinshelwood-Hougen-Watson (LHHW) model), no a priori chemical assumptions are made. The lumped molecular model is rather based on the reaction steps of the full microkinetic model; it has effective parameters that are directly linked to those of the full microkinetic model, and is mechanistically sound being able to capture important experimental observations, such as the 1st-order dependence on $\mathrm{CH}_{4}$ concentration, the independence on the coreactant $\left(\mathrm{H}_{2} \mathrm{O}\right)$ and the product inhibition at lower temperatures. In the case of the Ni catalyst, we use the empirical kinetic model of $\mathrm{Xu}$ and Froment (1989), which consists of LHHW type of reaction rate equations for the SR, WGS, and RM reactions, Eqs. (1-3).

SR : $\mathrm{CH}_{4}+\mathrm{H}_{2} \mathrm{O} \leftrightarrow \mathrm{CO}+3 \mathrm{H}_{2}, \quad \Delta H=+206 \mathrm{~kJ} / \mathrm{mol}$

WGS : $\mathrm{CO}+\mathrm{H}_{2} \mathrm{O} \leftrightarrow \mathrm{CO}_{2}+\mathrm{H}_{2}, \quad \Delta H=-41 \mathrm{~kJ} / \mathrm{mol}$

$\mathrm{RM}: \mathrm{CH}_{4}+2 \mathrm{H}_{2} \mathrm{O} \leftrightarrow \mathrm{CO}_{2}+4 \mathrm{H}_{2}, \quad \Delta H=+164 \mathrm{~kJ} / \mathrm{mol}$

A (surface area per mass) value of $4.1 \mathrm{~m}^{2} / \mathrm{g}$ is used to calculate the reaction rates for $\mathrm{Ni}$ on an area basis. This value corresponds to that of $\mathrm{Xu}$ and Froment and is close to the values of Trimm and Lam (1980) $\left(4.9 \mathrm{~m}^{2} / \mathrm{g}\right)$ and Numaguchi and Kikuchi (1988) $\left(3.6 \mathrm{~m}^{2} / \mathrm{g}\right)$, as reported by de Smet et al. (2001).

Catalytic methane combustion on Pt is modeled using the singlestep rate expression developed in Deshmukh and Vlachos (2007). This is also a product of a posteriori reduction of a full microkinetic model (Mhadeshwar, 2005) and is based on the rate determining step (RDS) of the process being the dissociative adsorption of methane on Pt. The global rate expression for methane can be applied to higher alkanes under the assumption that the RDS and the most abundant reactive intermediate (MARI) do not change. In Deshmukh and Vlachos (2007), kinetic parameters for the combustion of lower alkanes, ranging from methane to butane, on noble metals are provided. 
Therein, only the sticking coefficient and the activation energy for the fuel adsorption change. Adsorption activation energies of 10 and $4 \mathrm{kcal} / \mathrm{mol}$ for methane and propane combustion, respectively, are used in this work.

\section{Role of the combustible fuel: propane vs. methane}

In previous works (Deshmukh and Vlachos, 2005a, 2005b; Stefanidis and Vlachos, 2008; Stefanidis et al., 2009), we used propane as combustible fuel because of its higher stability compared to methane (Norton and Vlachos, 2004). In general, higher hydrocarbons have a lower activation energy compared to methane; they ignite at lower temperatures, are more resilient to heat losses, and offer a wider operating regime.

Our calculations have revealed that propane catalytic combustion in standalone microchannels is sustainable at heat losses or heat transfer twice as high as the critical heat loss for methane (not shown). In this work, we compare propane and methane catalytic combustion in the stable regime in terms of different operating lines away from the extinction or blowout stability limits. The inlet propane/air equivalence ratio is 0.85 . The inlet methane/air equivalence ratio is $\sim 0.91$. Using these inlet compositions, the heat release upon complete combustion is the same for the two combustible fuels at equal combustible mixture inlet velocities. Methane SR on Rh takes place in the reforming channel.

Fig. 2A presents the power output from the microreactor in terms of hydrogen and Fig. 2B the maximum wall temperature vs. combustible stream inlet velocity along different operating lines. The power output is calculated based on the lower heating value (LHV) of hydrogen at $300 \mathrm{~K}$. This graph is in essence a two-parameter continuation diagram where both combustible and reforming stream inlet velocities vary. In Fig. 2A, the SR stream inlet velocity is gradually increased from the bottom to the top of the graph for each combustible inlet velocity. The solid lines with filled symbols represent operating lines with propane as the combustible fuel, and the dashed lines with open symbols those with methane as the combustible fuel. Irrespective of the combustible fuel, three operating lines exist: (1) The materials stability line at low SR inlet velocities. This represents a maximum allowable temperature limit for the wall material, which is set to $1500 \mathrm{~K}$ following previous work (Deshmukh and Vlachos, 2005a, 2005b; Kaisare et al., 2008; Stefanidis et al., 2008; Stefanidis and Vlachos, 2008). (2) The breakthrough line, which delimits the complete conversion regime in the SR channel (methane conversion $\sim 99 \%$ ); underneath it (low SR flow rates), conversion is nearly complete and temperatures are high; above it, (high SR flow rates), conversion is incomplete and temperatures are lower. (3) The maximum power output line in the incomplete conversion regime, which corresponds to the maximum hydrogen yield, and is determined from the inlet flow rate and the methane conversion (Stefanidis et al., 2009). Depending on the overall process flow sheet, operation along the breakthrough line or the maximum power output line may be desirable.

Fig. $2 \mathrm{~A}$ shows that in the complete conversion regime, the materials stability lines for the two fuels practically coincide; the same is true for the breakthrough lines. This is because the high temperatures, for the residence times considered, allow for complete combustion of either fuels and complete conversion of methane in the SR channel. The same heat input upon complete combustion explains the approximately equal maximum temperatures along the two operating lines shown in Fig. 2B.

The combustible fuel plays an important role when the reactor is operated along the maximum power output line. Past a certain combustible inlet velocity, the maximum power output lines for the two fuels progressively diverge. In the incomplete conversion regime, temperatures are lower, and methane, which has higher activation

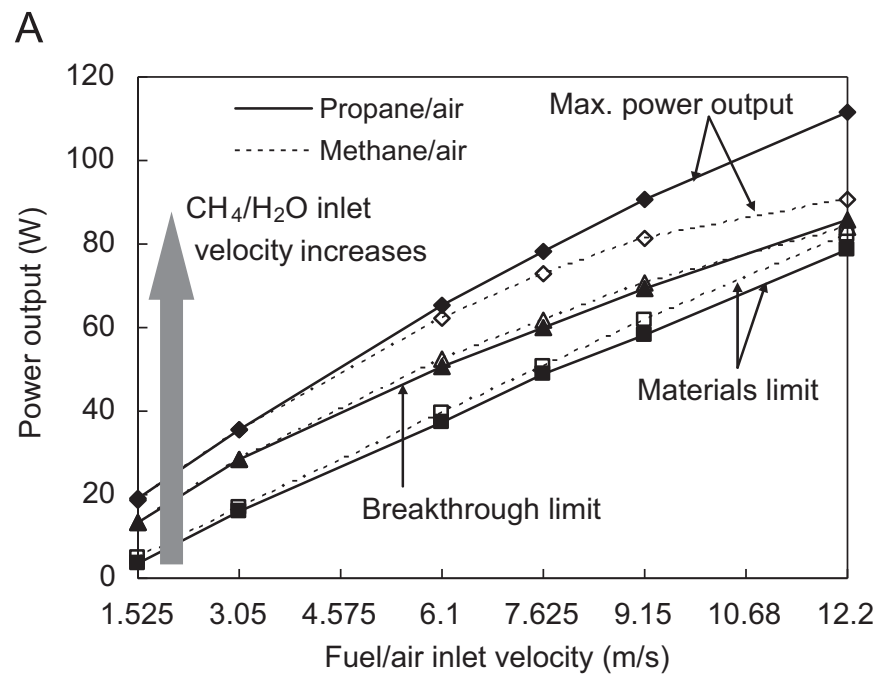

B

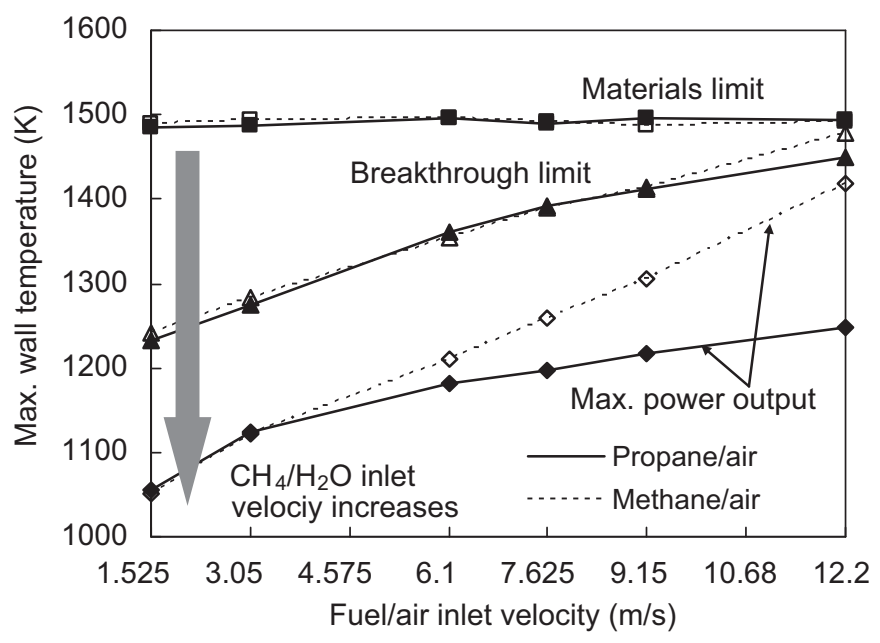

Fig. 2. (A) Power output and (B) maximum wall temperature vs. methane/air and propane/air inlet velocity along the operating lines of materials stability, methane breakthrough in the reforming channel, and maximum power output.

energy for adsorption than propane, becomes sensitive to temperature at low residence times (high combustible inlet velocities). Methane breakthrough in the combustion channel occurs at lower SR inlet velocities, compared to the ones at propane breakthrough. Therefore, the maximum power output in the case of methane decreases. At the maximum combustible inlet velocity considered in Fig. $2(12.2 \mathrm{~m} / \mathrm{s})$, the maximum power output with propane is $20 \mathrm{~W}$ higher than that with methane at $200 \mathrm{~K}$ lower temperature (Fig. 2B). At this point, the three operating lines in the case of methane are close to one another and practically determine the approximate upper bound of the combustible inlet velocity and the theoretical maximum power that can be obtained with this fuel. On the contrary, in the case of propane, there is a wide power output window at $12.2 \mathrm{~m} / \mathrm{s}$, i.e., further increase in the combustible inlet velocity along the maximum power output line is feasible. The conclusion from Fig. 2 is that use of higher hydrocarbons as combustible fuels in multifunctional reactors extends the inlet velocity operating regime and the theoretical maximum power (hydrogen yield) that can be obtained. Similar behavior is expected with other fast burning fuels, like $\mathrm{CO}$ and $\mathrm{H}_{2}$. 
A

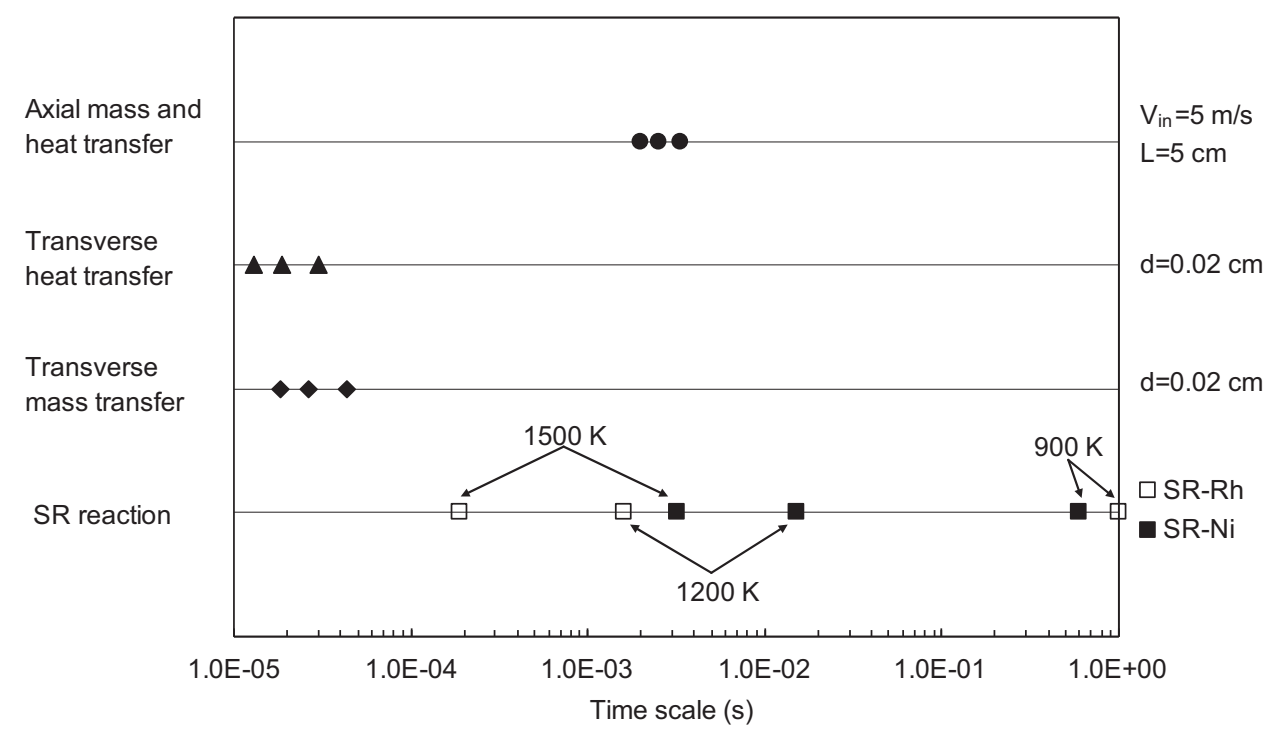

B

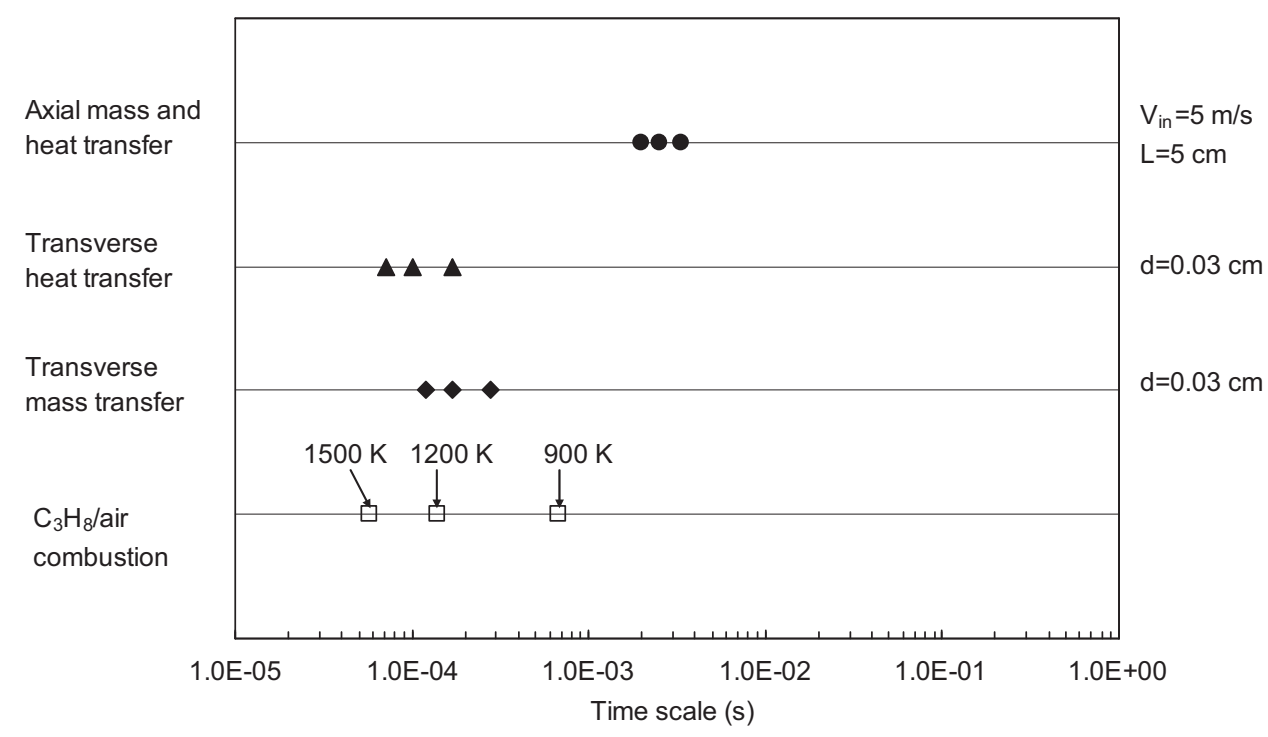

Fig. 3. Transport and reaction time scales in (A) the reforming channel and (B) the combustion channel. The leftmost, middle, and rightmost (transport relevant) points correspond to time scales at 1500,1200 , and $900 \mathrm{~K}$, respectively.

\section{Role of the reforming catalyst: rhodium vs. nickel}

Industrially, $\mathrm{SR}$ on $\mathrm{Ni}$ is a relatively bulky process with a typical time scale in the order of seconds (Farrauto et al., 2003). In Stefanidis and Vlachos (2008), Stefanidis et al. (2009), we showed that methane $\mathrm{SR}$ on $\mathrm{Rh}$ at microscale is feasible at millisecond contact times due to intrinsic fast chemistry and fast mass/heat transfer. Herein, we compare SR on $\mathrm{Rh}$ to $\mathrm{Ni}$ in order to explore whether miniaturization alone (fast transport) is sufficient for process intensification using $\mathrm{Ni}$. Below, a propane/air mixture at an equivalence ratio of 0.85 is used as the combustible stream.

Fig. 3 presents the relevant process time scales in the reforming channel for both $\mathrm{Rh}$ and $\mathrm{Ni}$ and in the combustion channel. The intrinsic reaction time scales are calculated as $C_{\text {fuel }} /\left(\right.$ Reaction rate $\left.\cdot \hat{a}_{\mathrm{g}}\right)$, where $\hat{a}_{\mathrm{g}}$ is the surface to volume ratio. They are computed at the three temperatures indicated and 50\% conversion of a reforming mixture with inlet $S: C=2: 1$ or a propane/air combustion mixture with an inlet equivalence ratio of 0.85 . The SR and combustion reaction rates are based on the kinetic models discussed in the model description. The reaction rates vary with position and composition; the numbers herein are representative order of magnitude.

In the reforming channel (Fig. 3A), both $\mathrm{Rh}$ and Ni SR kinetic models have been derived and validated at the lowest temperatures used herein; at higher temperatures, time scales are extrapolations. From 900 to $1500 \mathrm{~K}$, the reaction time scale on Rh varies from $\sim 1 \mathrm{~s}$ to $\sim 200 \mu \mathrm{s}$ and for Ni from $\sim 1 \mathrm{~s}$ to $\sim 1 \mathrm{~ms}$. The intrinsic speed on both catalysts is similar at low temperatures but Rh's speed surpasses Ni's at higher temperatures. Calculations at different compositions have shown that the ratio of SR time scale on Rh to SR time scale on $\mathrm{Ni}$ is up to 25 over the temperature range $900-1500 \mathrm{~K}$. At $\sim 1200 \mathrm{~K}$ and $50 \%$ conversion (representative microreactor conditions), SR on Rh appears roughly an order of magnitude faster than on $\mathrm{Ni}$.

Fig. $3 \mathrm{~A}$ also shows the transverse mass and heat transfer time scales being calculated as the inverse $d^{2} / 4 D$ and $d^{2} / 4 \alpha$, respectively, where $d$ is the gap size, $D$ is the methane diffusivity, and $\alpha$ is the mixture thermal conductivity. The transverse transport time scales are 


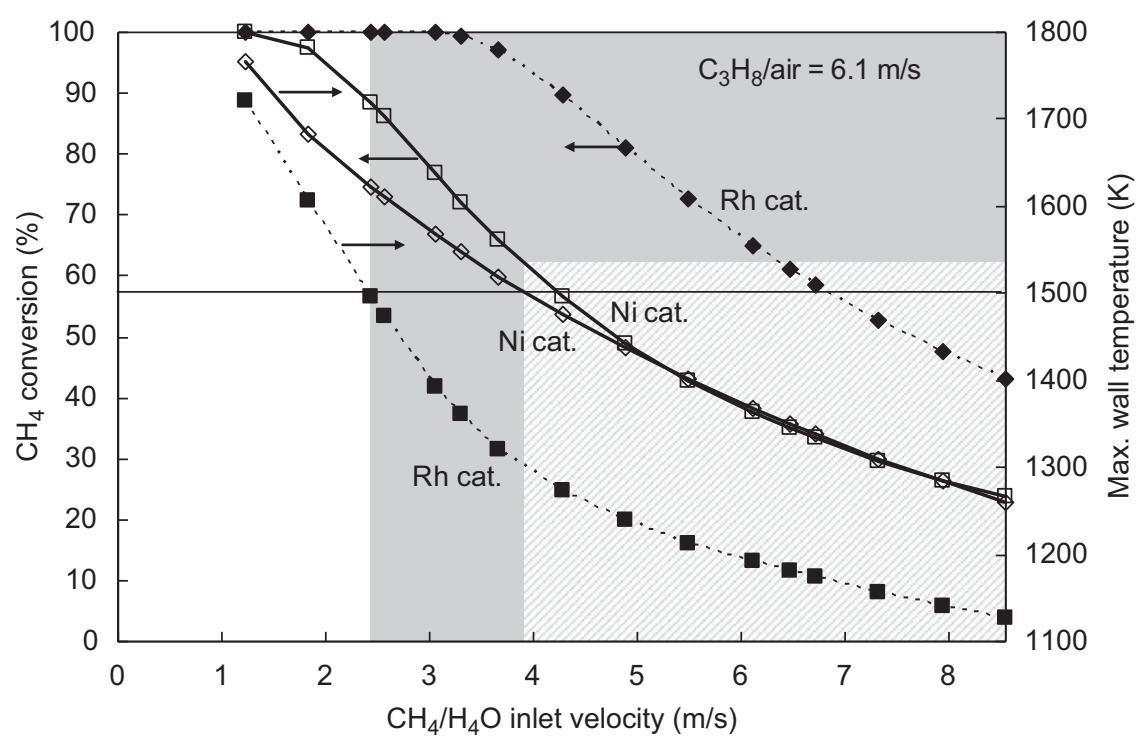

Fig. 4. Methane conversion in the reforming channel and maximum wall temperature vs. reforming stream inlet velocity at a constant propane/air inlet velocity of $6.1 \mathrm{~m} / \mathrm{s}$ in the combustion channel.

tens of microseconds for the temperature range and nominal gap size considered $(d=0.02 \mathrm{~cm})$; they are typically quite shorter than the reaction time scales. Finally, the average residence times determine axial mass/heat transfer for convection-dominated problems, as the one studied here. They are in the order of a few milliseconds (calculated based on a $5 \mathrm{~cm}$ long reactor and average velocities, using an inlet velocity of $5 \mathrm{~m} / \mathrm{s}$ corrected to the average temperature of 900 , 1200 , and $1500 \mathrm{~K}$ ). For most reactor conditions, the SR reaction time is lower than the residence time; however, as shown in Fig. 3, the reaction time scale can become comparable to or longer than the residence time (e.g., at lower temperatures, especially with $\mathrm{Ni}$ ), resulting in possible or even considerable methane breakthrough. Overall, the time scales indicate that process intensification, in terms of external heat and mass transfer in the SR reaction, has been achieved. Catalyst improvement (in loading or activity), with about an order of magnitude SR catalyst rate increase will be beneficial.

Fig. 3B is the counterpart of Fig. 3A for the combustion channel. The propane combustion time scales range from $\sim 60$ to $\sim 600 \mu$ s. They are shorter than the reforming time scales across the temperature range, with the difference being more pronounced at lower temperatures: at $900,1200,1500 \mathrm{~K}$, the intrinsic combustion time scale is faster than the reforming time scale on Rh (the values between parentheses indicate comparison with $\mathrm{Ni}$ ) by 3 (3) orders of magnitude, 1 (2) order(s) of magnitude, and 3 (56) times, respectively. Furthermore, the transverse transfer time scales in the combustion channel are slower than the corresponding time scales in the reforming channel since the gap size is higher and the thermal and fuel mass diffusivities lower. Clearly, unlike the reforming channel, the reaction and transverse mass transfer time scales now overlap, indicating that the combustion process operates in a mixed reactiontransport controlled regime. Consequently, the process can be further intensified via decreasing the combustion channel gap size. Catalyst improvement (in loading or activity) will not be as beneficial since the process will become transport controlled. No overlap between the residence times and the other time scales is obtained implying that the combustion process is less sensitive to breakthrough than the reforming process.

Fig. 4 compares the performance of $\mathrm{Rh}$ and $\mathrm{Ni}$ in the multifunctional microreactor (Fig. 1) in terms of methane conversion in the reforming channel and maximum wall temperature vs. reforming stream inlet velocity. Nominal catalyst loading is used in both channels. This is expressed via the surface area factor (SAF), which is the ratio of the 'effective' catalyst surface area to the geometric one. The nominal SAF for the combustion channel is set to 1.7, following Kaisare et al. (2008); the nominal SAF for the reforming channel is set to 1 . As explained in Kaisare et al. (2008), these values, which multiply the reaction rates, account for the uncertainties of the actual catalyst surface that is available for reaction as well as possible internal mass transfer limitations, catalyst sintering and deactivation. For noble metals deposited on anodized alumina wafers, SAF can be as high as 100 (Norton et al., 2006). Consequently, the values used in this work are rather conservative estimates. In general, higher SAF values make the process faster and allow for operation at higher flow rates, which entails higher power output and increased stability (Stefanidis and Vlachos, 2008; Kaisare et al., 2008). Decoupling of internal-mass transfer from surface area effects is entirely possible, but would depend on the catalyst (e.g., washcoat and wafer thickness, pore size, etc.), and is not explicitly done here. Fig. 4 shows that at low reforming stream inlet velocities, conversions and temperatures are high; both gradually decrease with increasing reforming stream inlet velocity. The horizontal line at $1500 \mathrm{~K}$ denotes the materials stability limit. The grey shaded and the hatched zones show the attainable methane conversion range at temperatures below the materials stability limit for $\mathrm{Rh}$ and $\mathrm{Ni}$, respectively. It is clear that using $\mathrm{Rh}$, complete conversion can be obtained over a certain inlet velocity range $(\sim 2.5-4 \mathrm{~m} / \mathrm{s})$ at temperatures below the materials stability limit (dashed lines). On the other hand, on Ni the methane conversion is only $\sim 60 \%$ at the materials stability limit (solid lines). Much higher (undesirable) temperatures are required to achieve complete conversion with nominal Ni loading by using lower reforming flow rates or increasing combustible flow rates. Mechanical stability will be though an important issue.

Our results indicate that reactor miniaturization alone is insufficient to render the traditionally limited heat transfer SR process on Ni workable for portable and distributed power devices. Under nominal (same as on $\mathrm{Rh}$ ) conditions, $\mathrm{Ni}$ is too slow to cope with the rate of heat release and temperatures get too high. This is because the intrinsic SR chemistry on Ni is rate limiting. Reactor intensification is not enough; a fast reforming catalyst is also necessary. Rh achieves precisely this. 
A

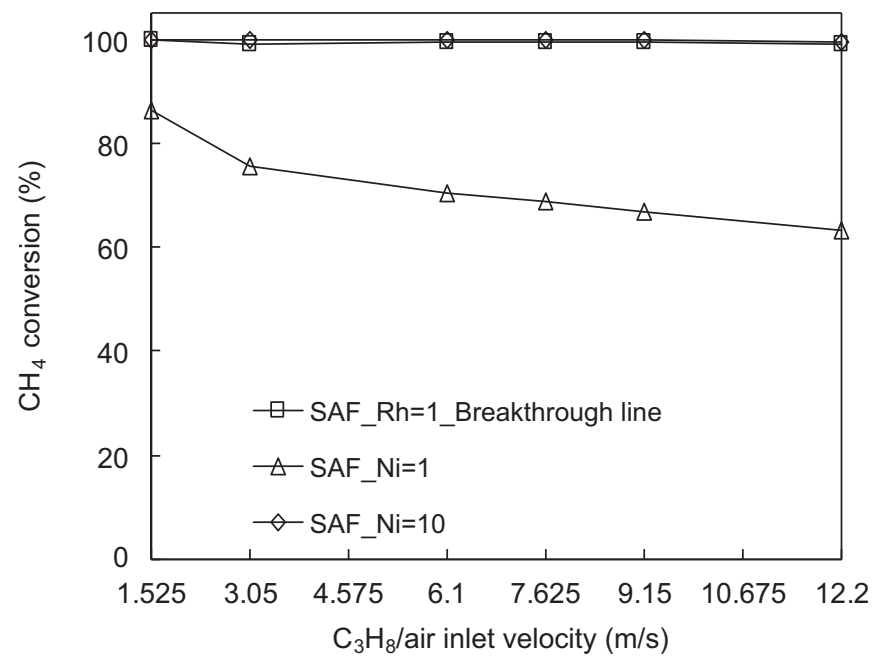

B

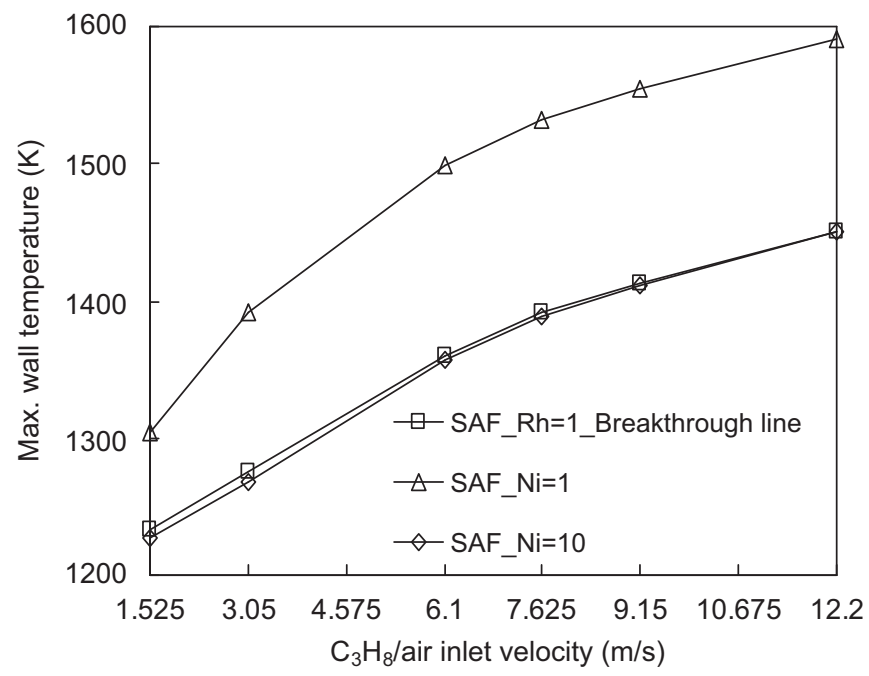

C

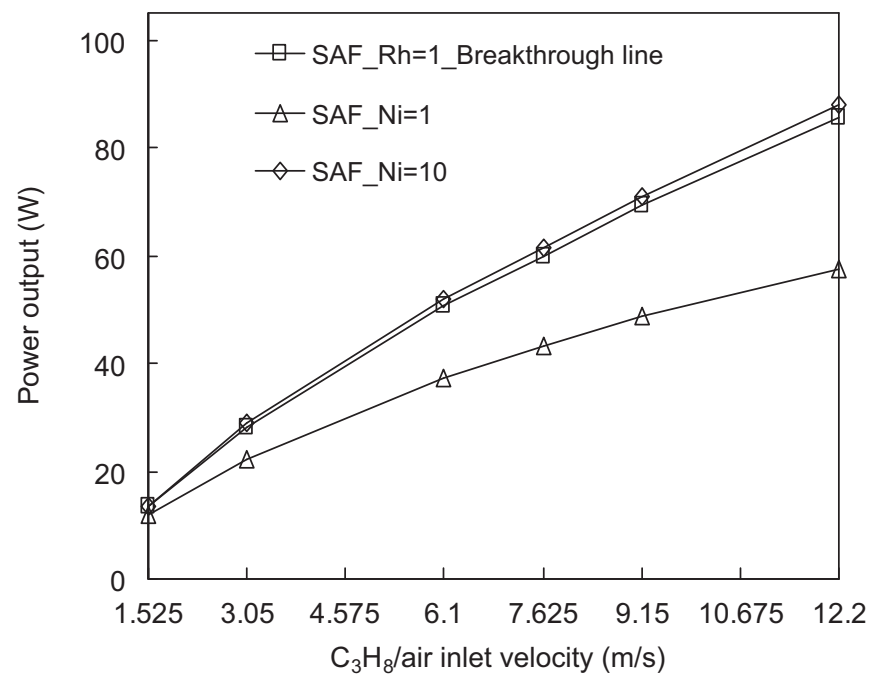

Fig. 5. (A) Methane conversion, (B) maximum wall temperature, and (C) power output vs. combustible stream inlet velocity. The breakthrough operating line for $\mathrm{Rh}$ with nominal catalyst loading $(\mathrm{SAF}=1)$ and for $\mathrm{Ni}$ with $\mathrm{SAF}=1$ and a tenfold increase in the Ni loading $(\mathrm{SAF}=10)$.
Fig. 5 presents the methane conversion, maximum wall temperature, and power output vs. combustible stream inlet velocity along the breakthrough line for $\mathrm{Rh}$ at nominal catalyst loading $(\mathrm{SAF}=1)$ and for $\mathrm{Ni}(\mathrm{SAF}=1$ and with a tenfold increase in the Ni loading $(\mathrm{SAF}=10)$ ). With nominal Ni loading, complete methane conversion is impossible (Fig. 5A), and maximum temperatures exceed the materials stability limit at higher velocities due to low methane conversion. On the other hand, when the Ni loading increases $(\mathrm{SAF}=10)$, almost identical reactor performance to that with $\mathrm{Rh}$ is obtained (Fig. 5A-C). This is in agreement with Fig. 3 showing that the Ni chemistry is roughly an order of magnitude slower than that of Rh under microreactor conditions. Increasing Ni loading, reducing nanoparticle size, and minimizing internal mass transfer limitations are possible routes to increasing Ni's SAF. While SAF as high as $\sim 100$ is feasible in microreactors, at least at lower temperatures, an obvious question is of course catalyst stability, i.e., how one can minimize sintering and evaporation at high temperatures to ensure high $\mathrm{SAF}$ for Ni and avoid coking. Due to these issues, it remains to be seen experimentally if microreformers running on $\mathrm{Ni}$ are possible.

Some alternative strategies for improving microreformers (reducing temperature, increasing conversion) running on $\mathrm{Ni}$ are summarized next (data not shown). Operating with a lower combustible flow rate or a higher reforming flow rate can reduce the maximum temperature. In this case, a longer reactor can give similar performance with Rh. In order to decrease the maximum temperature, the combustion channel gap size could be increased to make the process more transport limited and slow down the rate of heat release; however, breakthrough of propane occurs. A decrease of the combustion catalyst loading (SAF) up to $\sim 25$ times reduces the maximum temperature but methane SR conversion is only moderate. A longer reactor combined with a reduced combustion SAF may give complete fuel conversion at reduced temperatures. Overall, reducing the activity/loading of the combustion catalyst, increasing the corresponding one of the reforming catalyst, use of a lower combustible flow rate and a longer reactor could give results comparable to $\mathrm{Rh}$ with possibly about an order of magnitude less compact systems.

\section{Conclusions}

Simulations of a coupled combustion/reforming microdevice have been performed using methane and propane as the combustible fuel as well as $\mathrm{Rh}$ and $\mathrm{Ni}$ as the reforming catalyst. Their comparison has yielded the following conclusions:

- Use of propane as combustible fuel extends the (high) inlet velocity operating regime as well as the maximum power and hydrogen yield that can be obtained. In the lower hydrogen yield regime (lower steam reforming flow rates), fuel conversions are complete and no apparent differences between combustible fuels are found if the equivalence ratios are such that the same heat input is supplied.

- The difference in the SR reaction time scales for Rh and Ni highly depends on the reaction temperature and to a lesser extend on composition. As the temperature increases, the SR on Rh becomes faster than on $\mathrm{Ni}$ by $\sim 3-20$ over the temperature range $1000-1500 \mathrm{~K}$. At intermediate (microreactor relevant) temperatures, SR on Rh is approximated an order of magnitude faster than on $\mathrm{Ni}$.

- At the nominal reforming channel gap size, transverse transport time scales are shorter than reaction time scales. Process (heat and mass transfer) intensification is sufficient. However, the residence time and the reaction times may overlap for certain conditions (e.g., lower temperatures, less active catalysts than Rh). Operation at moderately high temperatures (e.g., $\sim 1200 \mathrm{~K}$ ) will be advantageous for having compact systems. 
- Process intensification via miniaturization is not sufficient for portable and distributed SR processing. Fast reforming catalysts are needed to remove the heat released via combustion. Otherwise, system temperatures are too high for practical operation. While temperature reduction is, in principle, possible via combustible flow rate reduction or fuel dilution, extinction of catalytic combustion may be unavoidable; longer residence times will be necessary for high conversions. Overall, catalyst and process intensification must be symbiotic.

- SR on Ni at microscale with millisecond contact times is in principle feasible at increased catalyst loadings. Suitable strategies can lead to high efficiency with at least an order of magnitude (most probably by two orders of magnitude to reduce the higher temperatures) increase in system size using $\mathrm{Ni}$ as the reforming catalyst. $\mathrm{Ni}$ thermal and poisoning stability may be an issue for practical realization of a Ni based SR process.

\section{Acknowledgment}

This work was supported in part by the NSF (CBET-0729714).

\section{References}

de Smet, C.R.H., de Croon, M.H.J.M., Berger, R.J., Marin, G.B., Schouten, J.C., 2001. Design of adiabatic fixed-bed reactors for the partial oxidation of methane to synthesis gas. Application to production of methanol and hydrogen-for-fuelcells. Chemical Engineering Science 56 (16), 4849-4861.

Deshmukh, S., Vlachos, D.G., 2007. A reduced mechanism for methane and one-step rate expressions for fuel-lean catalytic combustion of small alkanes on noble metals. Combustion and Flame 149 (4), 366-383.

Deshmukh, S.R., Vlachos, D.G., 2005a. CFD simulations of coupled, countercurrent combustor/reformer microdevices for hydrogen production. Industrial \& Engineering Chemistry Research 44 (14), 4982-4992.

Deshmukh, S.R., Vlachos, D.G., 2005b. Effect of flow configuration on the operation of coupled combustor/reformer microdevices for hydrogen production. Chemical Engineering Science 60 (21), 5718-5728.

Donazzi, A., Bereta, A., Groppi, G., Forzatti, P., 2008a. Catalytic partial oxidation of methane over a $4 \% \mathrm{Rh} /$ alpha- $\mathrm{Al}_{2} \mathrm{O}_{3}$ catalyst Part I: kinetic study in annular reactor. Journal of Catalysis 255 (2), 241-258.

Donazzi, A., Bereta, A., Groppi, G., Forzatti, P., 2008b. Catalytic partial oxidation of methane over a $4 \% \mathrm{Rh} /$ alpha- $\mathrm{Al}_{2} \mathrm{O}_{3}$ catalyst Part II: role of $\mathrm{CO}_{2}$ reforming. Journal of Catalysis 255 (2), 259-268.

Farrauto, R., Hwang, S., Shore, L., Ruettinger, W., Lampert, J., Giroux, T., Liu, Y. Ilinich, O., 2003. New material needs for hydrocarbon fuel processing: generating hydrogen for the PEM fuel cell. Annual Review of Materials Research 33, 1-27.

FLUENT 6.2, 2004. Fluent Inc.: Lebanon, NH.

Kaisare, N.S., Vlachos, D.G., 2007a. Extending the region of stable homogeneous micro-combustion through forced unsteady operation. Proceedings of the Combustion Institute 31 (2), 3293-3300.

Kaisare, N.S., Vlachos, D.G., 2007b. Optimal reactor dimensions for homogeneous combustion in small channels. Catalysis Today 120 (1), 96-106.

Kaisare, N.S., Deshmukh, S.R., Vlachos, D.G., 2008. Stability and performance of catalytic microreactors: simulations of propane catalytic combustion on Pt. Chemical Engineering Science 63 (4), 1098-1116.
Kolios, G., Glokler, B., Gritsch, A., Morillo, A., Eigenberger, G., 2005. Heat-integrated reactor concepts for hydrogen production by methane steam reforming. Fuel Cells 5, 52-65.

Kolios, G., Gritsch, A., Morillo, A., Tuttlies, U., Bernnat, J., Opferkuch, F., Eigenberger, G., 2007. Heat-integrated reactor concepts for catalytic reforming and automotive exhaust purification. Applied Catalysis B-Environmental 70 (1-4), 16-30.

Maestri, M., Vlachos, D.G., Bereta, A., Groppi, G., Tronconi, E., 2008. Steam and dry reforming of methane on Rh: microkinetic analysis and hierarchy of kinetic models. Journal of Catalysis 259 (2), 211-222.

Maestri, M., Vlachos, D.G., Bereta, A., Groppi, G., Tronconi, E., 2009. A C 1 microkinetic model for $\mathrm{CH}_{4}$ activation on $\mathrm{Rh} / \mathrm{Al}_{2} \mathrm{O}_{3}$. A.I.Ch.E Journal 55, 993-1008.

Mei, H., Li, C.Y., Ji, S.F., Liu, H., 2007. Modeling of a metal monolith catalytic reactor for methane steam reforming-combustion coupling. Chemical Engineering Science 62 (16), 4294-4303.

Mhadeshwar, A.B., 2005. A hierarchical multiscale approach for predictive microkinetic modeling of hydrogen production. Ph.D., Chemical Engineering, University of Delaware, Newark.

Mhadeshwar, A.B., Vlachos, D.G., 2005. Hierarchical multiscale mechanism development for methane partial oxidation and reforming, and for thermal decomposition of oxygenates on Rh. Journal of Physical Chemistry B 109, 16819-16835.

Norton, D.G., Vlachos, D.G., 2004. A CFD study for propane/air microflame stability. Combustion and Flame 138, 97-107.

Norton, D.G., Deshmukh, S.R., Wetzel, E.D., Vlachos, D.G., 2005. Downsizing chemical processes for portable hydrogen production. In: Wang, Y., Holladay, J.D. (Eds.), Microreactor Technology and Process Intensification. ACS, New York, pp. 179-193.

Norton, D.G., Wetzel, E.D., Vlachos, D.G., 2006. Thermal management in catalytic microreactors. Industrial \& Engineering Chemistry Research 45 (1), 76-84.

Numaguchi, T., Kikuchi, K., 1988. Intrinsic kinetics and design simulation in a complex-reaction network-steam-methane reforming. Chemical Engineering Science 43 (8), 2295-2301.

Robbins, F.A., Zhu, H., Jackson, G.S., 2003. Transient modeling of combined catalytic combustion $/ \mathrm{CH}_{4}$ steam reforming. Catalysis Today 83, 141-156.

Stefanidis, G.D., Kaisare, N.S., Maestri, M., Vlachos, D.G., 2009. Methane steam reforming at microscales: operation strategies for variable power output at millisecond contact times. A.I.Ch.E. Journal 55 (1), 180-191.

Stefanidis, G.D., Kaisare, N.S., Vlachos, D.G., 2008. Modeling ignition in catalytic microreactors. Chemical Engineering \& Technology 31 (8), 1170-1175.

Stefanidis, G.D., Vlachos, D.G., 2008. Millisecond methane steam reforming via process and catalyst intensification. Chemical Engineering \& Technology 31 (8), 1201-1209.

Tonkovich, A.Y., Yang, B., Perry, S.T., Fitzgerald, S.P., Wang, Y., 2007. From seconds to milliseconds to microseconds through tailored microchannel reactor design of a steam methane reformer. Catalysis Today 120, 21-29.

Trimm, D.L., Lam, C.-W., 1980. The combustion of methane on platinum-alumina fibre catalysts-I Kinetics and mechanism. Chemical Engineering Science 35, $1405-1413$.

Trimm, D.L., Onsan, Z.I., 2001. Onboard fuel conversion for hydrogen-fuel-cell-driven vehicles. Catalysis Reviews 43 (1\&2), 31-84.

Venkataraman, K., Wanat, E.C., Schmidt, L.D., 2003. Steam reforming of methane and water-gas shift in catalytic wall reactors. A.I.Ch.E. Journal 49 (5), 1277-1284.

Vlachos, D.G., 2008. In: Barton, P.I., Mitsos, A. (Eds.), Microreaction Engineering: Processes, Detailed design and Modeling. Wiley, New York.

Xu, J.G., Froment, G.F., 1989. Methane steam reforming, methanation and water-gas shift .1. Intrinsic kinetics. A.I.Ch.E. Journal 35 (1), 88-96.

Yuan, J.L., Ren, F., Sunden, B., 2007. Analysis of chemical-reaction-coupled mass and heat transport phenomena in a methane reformer duct for PEMFCs. International Journal of Heat and Mass Transfer 50 (3-4), 687-701.

Zanfir, M., Gavriilidis, A., 2003. Catalytic combustion assisted methane steam reforming in a catalytic plate reactor. Chemical Engineering Science 58 (17), 3947-3960. 